January 1996

SNUTP 95-103

revised September 1996

Brown-HET-1016

hep-ph/9601336

ULG-PNT-95-1-JRC

\title{
BOUNDS ON THE SOFT POMERON INTERCEPT
}

\author{
Jean-René Cudell \\ Inst. de Physique, U. de Liège, Bât. B-5, Sart Tilman, B4000 Liège, Belgium \\ cudell@gw.unipc.ulg.ac.be \\ Kyungsik Kang円 \\ Physics Department, Brown University, Providence, RI 02912, U.S.A. \\ kang@het.brown.edu \\ and \\ Sung Ku Kimf \\ Dept. of Physics, Ewha Women's University, Seoul 120-750, Korea \\ skkim@mm.ewha.ac.kr
}

\begin{abstract}
The Donnachie-Landshoff fit of total cross sections has now become a standard reference for models of total, elastic and diffractive cross-sections. Adopting their philosophy that simple-pole exchanges should account for all data to present energies, we assess the uncertainties on their fits. Our best estimate for the pomeron intercept is $1.096_{-0.009}^{+0.012}$, but several models have a good $\chi^{2}$ for intercepts in the range $[1.07,1.11]$.
\end{abstract}

\footnotetext{
${ }^{1}$ supported in part by the USDOE contract DE-FG02-91ER 40688-Task A.

${ }^{2}$ supported in part by the Korea Science and Engineering Foundation through the Center for Theoretical Physics, Seoul National University, and by the Ministry of Education through contract BSRI-95-2427.
} 
In Regge theory, the energy dependence of total and elastic cross sections is implied by the analytic structure of the hadronic amplitude. The simplest singularities are poles in the $J$-plane, which correspond to the existence of bound states of mass $M$ and spin $J$ with $J=\alpha\left(M^{2}\right)$. The sum of the exchanges of all the particles on a trajectory $\alpha\left(M^{2}\right)$ leads to a hadronic amplitude which behaves, for $s \rightarrow \infty$ and $t=M^{2}<0$ finite, as:

$$
\mathcal{A}(s, t)=C s^{\alpha\left(M^{2}=t\right)} \pm(s \rightarrow-s)
$$

The last term, which arises from $s$ to $u$ crossing, is usually included as a Regge signature factor. Its sign depends on the charge parity of the exchange.

Meson trajectories are observed to be linear, as can be understood in a string model:

$$
\alpha\left(M^{2}\right)=\alpha_{0}+\alpha^{\prime} M^{2}
$$

with $\alpha_{0}$ the intercept, and $\alpha^{\prime}$ the slope of the trajectory. In particular, total cross sections are related to $\operatorname{Im\mathcal {A}}\left(s, t=M^{2}=0\right)$ by the optical theorem and involve only the intercept of the trajectory. Many such trajectories are present, but at high $s$ only the highest-spin trajectories will contribute: the others are suppressed by a power of $s$. Hence, from the observed meson spectrum, one expects total cross section to fall like $1 / \sqrt{s}$, as the leading meson trajectories are those of the $\rho, \omega, a$ and $f$ mesons, with $\alpha_{0} \approx 0.5$.

The fact that total cross sections rise at high energy forces one to introduce one further ingredient: the pomeron, which in its simplest version is another Regge trajectory, with an intercept slightly greater than 1 . This object has been hypothesized a long time ago [1], and presumably arises from the gauge sector of QCD. Its intercept and slope are then fundamental numbers characterizing the pure gauge sector of Yang-Mills SU(3). They control both the large- $s$ and the small- $x$ limits of hadronic cross sections.

There has been a renewed interest in this object as the range of $s$ and $1 / x$ has been expanding, at HERA and at the Tevatron, where e.g. rapidity gaps in deep inelastic scattering have been observed [2]. The situation at HERA is particularly confusing, as the pomeron intercept seems to vary with the scale of the problem, and with the masses of external particles: whereas it seems to be of the order of 1.1 in the total $\gamma p$ cross section, it increases slightly in quasi-elastic production of $\rho$ mesons, reaches values of the order of 1.25 in $J / \psi$ photoproduction, and increases further in high- $Q^{2}$ deep-inelastic 
scattering. At the same time, the gap cross section remain compatible with a low value of the intercept, as measured in the pomeron flux.

The only object which is well defined operationally is the soft intercept, i.e. the one which controls total cross sections at high-energy. How well that number is known is thus a central issue for HERA, and will no doubt have a bearing on the extrapolation of soft cross sections to LHC energies. Hence we propose in this letter to re-evaluate the simple-pole parametrisation and to estimate the errors on the various parameters.

The information on the soft intercept comes from total cross sections. As the pomeron controls the high-energy behaviour of the cross section, it will be most sensitive to the highest energy (i.e. $p p$ and $\bar{p} p$ ) data. There is one caveat to this, as one expects the model to break down at low energy (because of the presence of sub-leading trajectories) and at high-energy (because multiple pomeron exchanges must unitarise the cross sections to make them agree with the Froissart-Martin bound). Hence one must not only determine the best parameters, but also the range of validity of the model. One might think of resorting to a $\chi^{2}$ test in order to do this. This is not possible directly because of the low quality of the data. Indeed, the largest compilation of data, made available by the Particle Data Group [3] contains points which are inconsistent at the $16 \sigma$ level! [Note that this point is not shown any longer in the 1996 version of the particle data book, although it is still present in the compilation of data].

To illustrate these problems and their proposed solution, we first examine the Donnachie-Landshoff (DL) model in its original form [4]. This model fits the $p p$ and $\bar{p} p$ cross sections using a minimal number of trajectories. The higher meson trajectories, $a / f(C=+1)$ and $\rho / \omega(C=-1)$, are assumed to be degenerate, and their contributions are added to a pomeron term in the amplitude. The data are fitted for $\sqrt{s}>10 \mathrm{GeV}$, as lower trajectories would then contribute less than $1 \%$, which is less than the errors on the data. The result of their fit [5] is a pomeron intercept of 1.0808, for which they did not quote a $\chi^{2}$ or error bars, but simply mentioned that the $\chi^{2}$ was very flat near the minimum.

We show in the first column of Table 1 our results for such a fit. We use the usual definition

$$
\chi^{2}=\sum_{i}\left(\frac{\left.d_{i}-s_{i}^{-1} \operatorname{Im} \mathcal{A}\left(s_{i}, 0\right)\right)}{e_{i}}\right)^{2}
$$


with $d_{i} \pm e_{i}$ the measured $p p$ or $\bar{p} p$ total cross section at energy $\sqrt{s_{i}}$, and

$$
\operatorname{Im} \mathcal{A}(s, 0)=C_{-} s^{\alpha_{m}}+C_{+} s^{\alpha_{m}}+C_{P} s^{\alpha_{P}}
$$

where $C_{-}$flips sign when going from $p p$ to $\bar{p} p$. We shall use the notation $\alpha_{P}=1+\epsilon$ in the following.

\begin{tabular}{|c|c|c|c|}
\hline parameter & all data & filtered data $(2 \sigma)$ & filtered data $(1 \sigma)$ \\
\hline$\chi^{2}$ & 410.8 & 80.3 & 32.4 \\
$\chi^{2}$ per d.o.f. & 3.16 & 0.62 & 0.25 \\
\hline pomeron intercept-1 & $0.0912_{-0.0070}^{+0.0077}$ & $0.0887_{-0.0071}^{+0.0079}$ & $0.0863_{-0.0084}^{+0.0096}$ \\
pomeron coupling $(\mathrm{mb})$ & $19.3_{-1.7}^{+1.5}$ & $19.8_{-1.7}^{+1.6}$ & $20.4_{-2.1}^{+1.8}$ \\
\hline$\rho / \omega / a / f$ intercept-1 & $-0.382_{-0.071}^{+0.065}$ & $-0.373_{-0.073}^{+0.067}$ & $-0.398_{-0.090}^{+0.0083}$ \\
$\rho / \omega$ coupling $C_{-}(p p)(\mathrm{mb})$ & $-13.2_{-6.5}^{+4.1}$ & $-13.3_{-6.9}^{+4.3}$ & $-15.3_{-10.1}^{+5.7}$ \\
$a / f$ coupling $(\mathrm{mb})$ & $69_{-13}^{+20}$ & $62_{-12}^{+19}$ & $67_{-16}^{+25}$ \\
\hline
\end{tabular}

Table 1: Simple pole fits to total $p p$ and $\bar{p} p$ cross sections, assuming degenerate $C=+1$ and $C=-1$ exchanges.

The value of the $\chi^{2}=410$ for 135 data points and 5 parameters is totally unacceptable, as it corresponds to a confidence level (CL) of $2 \times 10^{-36}$ ! This CL gives the probability that the model with fixed parameters could generate the data through a random fluctuation. Note that all other fits have so far suffered from the same problem [8]. There are two possible outcomes to such a high value of the $\chi^{2}$ : either the model is to be rejected, or some of the data are wrong. As we already mentioned, there are a few obviously wrong points within the data. Hence before reaching conclusions about the model, one needs to tackle the issue of eliminating those points.

We propose here a reasonable criterion, which will give us the order of magnitude of the uncertainties. The best selection criterion would based on physics arguments to decide which experiments got the wrong results. Unfortunately, besides experimental questions, this would involve some personal bias, and prove infeasible for old (ISR) data, where most of the incompatibilities lie. Here, we propose the following approach, which is independent of any underlying theoretical model. Most data are bunched in several energy intervals. We ask that a given data point should not deviate by more than 1 or $2 \sigma$ from the average of all data in a bin of $\pm 1 \mathrm{GeV}$ centered around it (note that filtering at the $1 \sigma$ level rejects both the E710 and the CDF 
measurements, whereas both are kept when filtering at the $2 \sigma$ level ). All the data which do not fulfill this criterion are to be rejected. This eliminates some of the pathological points in a fit-independent manner, and brings in a dramatic improvement in the $\chi^{2} / d o f$., which falls down to less than 1 for the best fits. Note that this procedure is not unlike the one followed by UA4/2 in [6]. The number of standard deviations, as well as the width of the intervals considered are of course somewhat arbitrary. Because the data are concentrated at certain energies, the width does not matter too much, but one could certainly change the filtering to an arbitrary number of standard deviations. When considering large numbers of points, it is in fact quite likely that about one third will deviate by more than one standard deviation. Hence the $1 \sigma$ filtering is presumably too stringent, although we shall see that the results are stable when going from one selection criterion to the other.

We give in Table 2 the number of points kept when filtering the data, the full data sets being available at http://nuclth02.phys.ulg.ac.be/DATA.htm]. Note that in the following we shall also consider an alternative data set [0]. Its main difference with that of the Particle Data Group is that the statistical and systematic errors have been added in quadrature, and that it includes the measurements of the $\rho$ parameter.

\begin{tabular}{|c|c|c|c|c|}
\hline data set & $\sigma_{\text {tot }}^{p p}(\mathrm{mb})$ & $\sigma_{\text {tot }}^{p p}(\mathrm{mb})$ & $\rho^{p p}$ & $\rho^{\bar{p} p}$ \\
\hline P.D.G. [3] & 94 & 28 & - & - \\
P.D.G. [3] - $2 \sigma$ & 84 & 28 & - & - \\
P.D.G. [3] - $1 \sigma$ & 65 & 20 & - & - \\
Ref. [7] & 66 & 29 & 41 & 13 \\
Ref. [7] - $2 \sigma$ & 60 & 28 & 38 & 13 \\
Ref. [7]- $1 \sigma$ & 53 & 19 & 31 & 13 \\
\hline
\end{tabular}

Table 2: The number of points kept after data selection, for $\sqrt{s}>10 \mathrm{GeV}$.

If we now go back to the first line of Table 1, we see that the elimination of a handful of points drastically changes the $\chi^{2}$, and makes the model entirely acceptable. We can now proceed to evaluate the errors on the $\mathrm{N}$ parameters [9, 10, 11]. Here the data are given, and we are asking what is the effect of a random change of parameters. The value of $\chi^{2}-\chi_{\min }^{2}$ is distributed as a $\chi^{2}$ distribution with $\mathrm{N}$ parameters. We choose here to quote the intervals corresponding to the projection of the constant $\chi^{2}$ hypersurface containing 
$70 \%$ of the probability. This corresponds to $\chi^{2}=\chi_{\min }^{2}+6.06$ in the DL case with 5 parameters. Note that we do not trust the validity of the method used by the Particle Data Group in their fit to the DL model, as they simply renormalise the $\chi^{2}$ to $\chi^{2} / d o f .=1$ and let the new $\chi^{2}$ vary by one unit.

An important test of the data filtering method is that the central values and their errors should not depend too much on the filtering itself. Table 2 shows that this is indeed the case for our fit. We see that the pomeron intercept is determined to be about 1.090, and that it could be as high as 1.096. Filtering the data does change the value of $\chi_{\min }^{2}$, but does not affect its variation around the minimum too much. Hence the stability of the parameter values seems a more reasonable criterion than the value of $\chi_{\min }^{2}$.

We can now tackle the question of the energy range of validity of the model. The two basic requirements are that the $\chi^{2} / d o f$. be of the order of 1 , and that the determination of the intercept be stable. We show in Fig. 1 the result of varying the energy range. Clearly, the lower trajectories seem to matter for $\sqrt{s}_{\text {min }}<10 \mathrm{GeV}$, whereas the upper energy does not seem to modify the results (in other words, there is no sign of the onset of unitarisation). Hence we adopt $\sqrt{s}_{\text {min }}=10 \mathrm{GeV}$ as the lowest energy at which the model is correct. This happens to be the point at which $\chi_{\min }^{2}$ is lowest, too. This dependence on the lower energy cut explains why both the Particle Data Group [9] and Bueno and Velasco [12] obtain an wrong value for the intercept, much lower than ours.
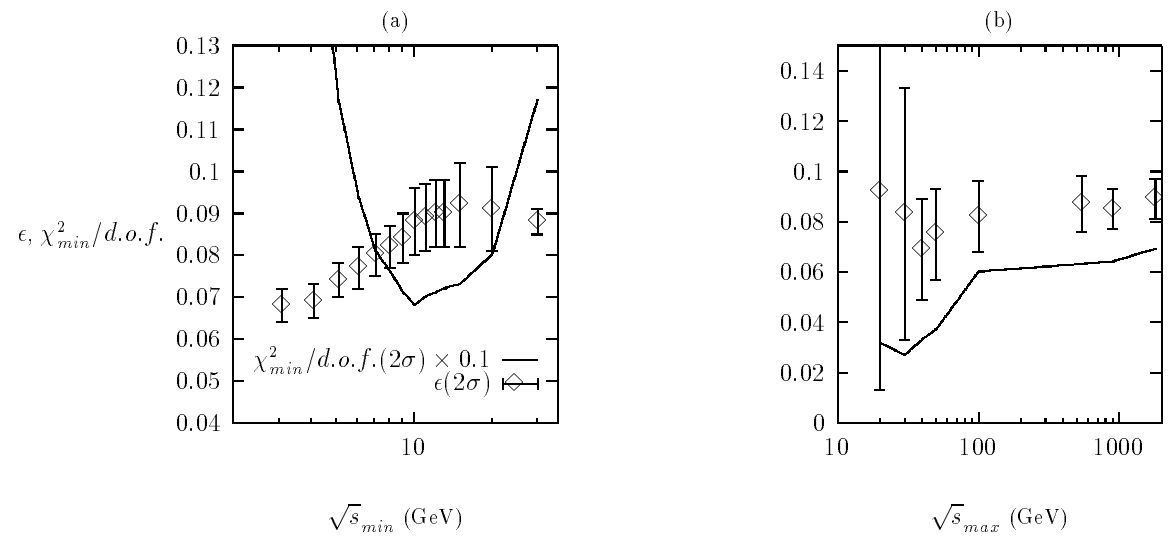

Figure 1: DL intercept-1 as a function of the lower (a) and upper (b) energy cuts on the data. The curve shows the $\chi^{2} /$ dof. for data filtered at the $2 \sigma$ level. 
One must wonder if it is possible to get a better determination of the soft pomeron intercept by using more data. After performing their fit on $p p$ and $\bar{p} p$ cross section, DL extended it to include all measured hadronic total cross sections. They found that this did not affect the value of the intercept very much. This is due to the fact that the other total cross sections have large errors, and that new parameters are introduced (namely the couplings of the Regge trajectories) each time one considers a new type of cross section. The Particle Data Group (PDG) [9] obtained very narrow determinations of the pomeron intercept from the other hadronic reactions. We believe that their conclusions are wrong, and illustrate this in the case of the $\pi^{ \pm} p$ total cross sections, for which they use $\sqrt{s}_{\text {min }} \approx 4 \mathrm{GeV}$ and obtain an intercept of $1.079 \pm 0.003$.
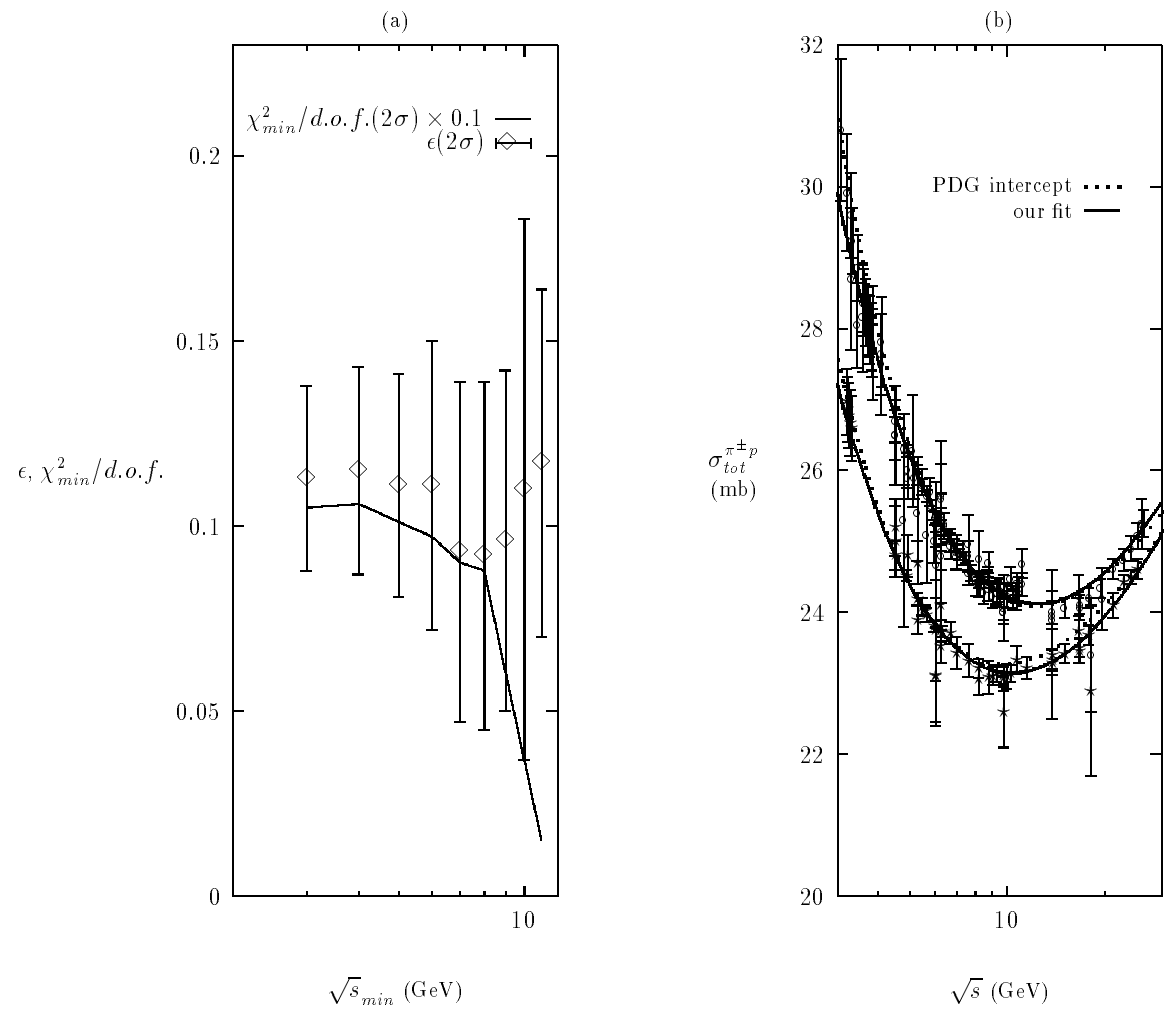

Figure 2: Pomeron intercept from $\pi p$ data: (a) shows the values of the intercept as the lower energy cut on the data is changed; (b) shows our best fit together with that of the Particle Data Group, together with the set of data filtered at the $2 \sigma$ level. 
We show in Fig. 2 our results for such a fit: for data filtered at the $2 \sigma$ level (92 points), and for $\sqrt{s}_{\min }=4 \mathrm{GeV}$, we obtain $\alpha_{0}=1.115_{-0.023}^{+0.030}$. There is no significant change if we modify the lower energy cutoff on the data. We show in Fig. (2.b) our best fit together with that of the Particle Data Group. Although according to their estimate our central value for the intercept is 10 standard deviations from theirs, we see that the two fits are indistinguishable. Hence we believe that both their standard deviations and their central values are wrong. These conclusions are not affected by the use of the full data set instead of the one filtered at the $2 \sigma$ level. The above value of the intercept in fact gives a slightly smaller $\chi^{2} /$ dof than the one quoted in the Particle Data Book. Note that this intercept is consistent with the one we got from the analysis of $p p$ and $\bar{p} p$ total cross sections. The conclusion from this exercise is that the errors from the low-energy hadronic data are large, especially if we use a low-energy cut-off of the order of 10 . Hence we want to limit ourselves to $p p$ and $\bar{p} p$ amplitudes at $t=0$.

\begin{tabular}{|c|c|c|c|}
\hline parameter & all data & filtered data $(2 \sigma)$ & filtered data $(1 \sigma)$ \\
\hline$\chi^{2}$ & 561.3 & 168.3 & 94.9 \\
$\chi^{2}$ per d.o.f. & 3.28 & 1.07 & 0.77 \\
\hline pomeron intercept-1 & $0.0840 \pm 0.0050$ & $0.0817_{-0.0053}^{+0.0055}$ & $0.0804_{-0.0061}^{+0.0064}$ \\
pomeron coupling $(\mathrm{mb})$ & $20.8 \pm 1.1$ & $21.4 \pm 1.1$ & $21.8 \pm 1.3$ \\
\hline$\rho / \omega / a / f$ intercept-1 & $-0.408_{-0.033}^{+0.032}$ & $-0.421_{-0.036}^{+0.034}$ & $-0.431_{-0.040}^{+0.037}$ \\
$\rho / \omega$ coupling $C_{-}(p p)(\mathrm{mb})$ & $-14.0_{-3.3}^{+2.6}$ & $-16.5_{-4.2}^{+3.3}$ & $-17.7_{-4.9}^{+3.7}$ \\
$a / f$ coupling $(\mathrm{mb})$ & $67.0_{-6.7}^{+7.6}$ & $66.6_{-7.2}^{+8.3}$ & $67.6_{-7.8}^{+9.0}$ \\
\hline
\end{tabular}

Table 3: Simple pole fit to total $p p$ and $\bar{p} p$ cross sections, and to the $\rho$ parameter, assuming degenerate $C=+1$ and $C=-1$ meson exchanges.

One more piece of data can be used however: the knowledge of the intercept is sufficient to determine the value of the real part of the amplitude, using crossing symmetry, and hence the measurements of the $\rho$ parameter provide an extra constraint. We use the data collected in Ref. [7], and obtain a somewhat worse fit, shown in Table 3, even when filtering data at the 1 or $2 \sigma$ level. For the $2 \sigma$ filtering of the data, the confidence level goes from $99.4 \%$ to $36 \%$. We show the curves corresponding to the second column of Table 3 in Fig. 3 with dotted lines. Whether one should worry about this, 
and about the change of central value for the parameters, is a matter of taste. Note however that it is this small change of central values, combined with the effect of too low an energy cut, that lead Bueno and Velasco [12] to conclude that simple-pole parametrisations were disfavored.
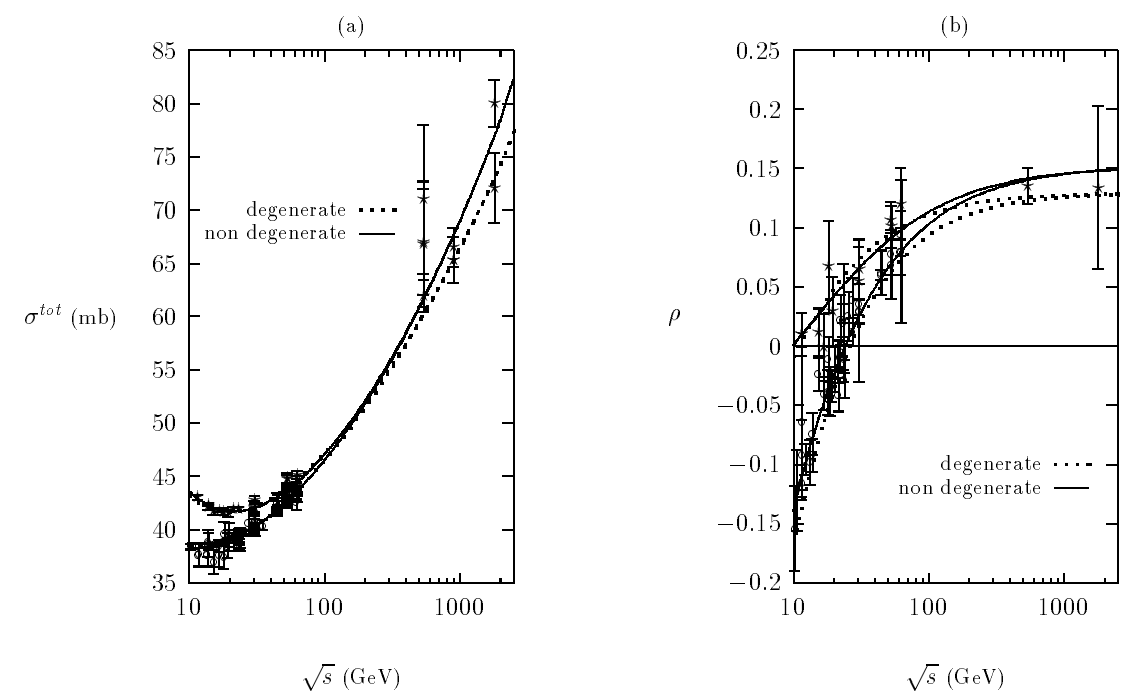

Figure 3: Best fits to $2 \sigma$ filtered data. The dotted lines correspond to the original DL model given in Eq. (舟), whereas the plain ones correspond to a model where the degeneracy of the lower trajectories is lifted, as in Eq. (6). The data points are the PDG data filtered at the $2 \sigma$ level.

Before concluding on the best value of the intercept, we need to examine the influence of low energy physics on the determination of the intercept. Although the energy cut $\sqrt{s}_{\text {min }}$ eliminates sub-leading meson trajectories, there is still an ambiguity in the treatment of the leading meson trajectories. In fact, we shall now see that a slightly different treatment to that of DL leads to a better $\chi^{2}$ and to more stable parameters. Indeed, there is no reason to assume that the $\rho, \omega, f$ and $a$ trajectories are degenerate. We show the data for the meson mass spectrum [9] in Fig. 4, as well as the separate best fits. 


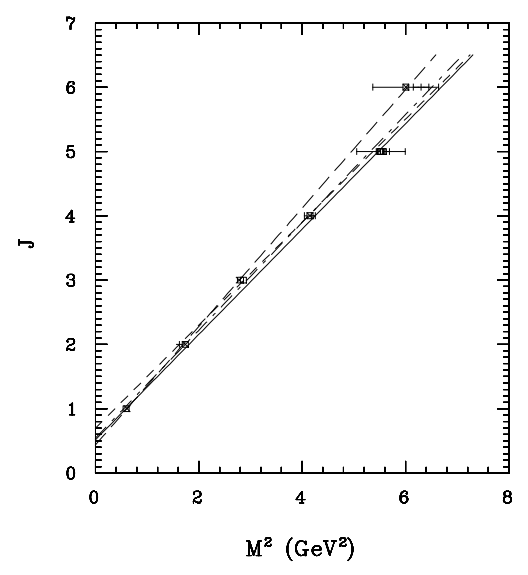

Figure 4: Best fit to the lower trajectories.

The trajectories plotted correspond to the following equations:

$$
\begin{aligned}
& \alpha_{\omega}(t)=(0.3467 \pm 0.0043)+(0.9213 \pm 0.0071) t \\
& \alpha_{\rho}(t)=(0.5154 \pm 0.0014)+(0.8198 \pm 0.0014) t \\
& \alpha_{a}(t)=(0.541 \pm 0.061)+(0.839 \pm 0.035) t \\
& \alpha_{f}(t)=(0.697 \pm 0.021)+(0.801 \pm 0.012) t
\end{aligned}
$$

At this point, it is tempting to use the central values of Eq. (5) for the lower trajectories. Such a procedure produces a a pomeron intercept of 1.095 with acceptable $\chi^{2}$. However, such a procedure is not satisfactory because it excludes the possibility of a mixing of the $f$ and the pomeron, or of the simultaneous exchange of several trajectories.

On the other hand, the data are not constraining enough to determine the effective intercepts of the four meson trajectories together with the pomeron intercept. We adopt an intermediate approach, which is to assume the exchange of separate + and - trajectories with independent intercepts:

$$
\operatorname{Im} \mathcal{A}(s, 0)=C_{-} s^{\alpha_{-}}+C_{+} s^{\alpha_{+}}+C_{P} s^{\alpha_{P}}
$$

The resulting numbers are shown in Table 4, and are plotted in Fig. 3 with plain lines. The $\chi^{2}$ is smaller than previously, and the parameters are more stable. The bounds on the soft pomeron intercept hardly depend on the criterion used to filter the data, and intercepts as large as 1.108 are allowed. 


\begin{tabular}{|c|c|c|c|}
\hline parameter & all data & filtered data $(2 \sigma)$ & filtered data $(1 \sigma)$ \\
\hline$\chi^{2}$ & 505.4 & 119.6 & 57.6 \\
$\chi^{2}$ per d.o.f. & 2.99 & 0.77 & 0.47 \\
\hline pomeron intercept-1 & $0.0990_{-0.0088}^{+0.0099}$ & $0.0964_{-0.0091}^{+0.0115}$ & $0.095_{+0.010}^{-0.013}$ \\
pomeron coupling $(\mathrm{mb})$ & $17.5_{-2.0}^{+1.9}$ & $18.0_{-2.2}^{+2.0}$ & $18.2_{-2.6}^{+2.3}$ \\
\hline$\rho / \omega$ intercept-1 & $-0.494_{-0.066}^{+0.056}$ & $-0.498_{-0.067}^{+0.057}$ & $-0.510_{-0.077}^{+0.064}$ \\
$\rho / \omega$ coupling $C_{-}(p p)(\mathrm{mb})$ & $-24.0_{-10.9}^{+6.8}$ & $-26.5_{-12.5}^{+7.7}$ & $-28.2_{-15.4}^{+8.9}$ \\
\hline$a / f$ intercept-1 & $-0.312_{-0.052}^{+0.051}$ & $-0.315 \pm 0.058$ & $-0.324 \pm 0.066$ \\
$a / f$ coupling $(\mathrm{mb})$ & $56.8_{-6.7}^{+8.1}$ & $54.9_{-7.2}^{+9.0}$ & $56.2_{-7.8}^{+9.9}$ \\
\hline$\sigma_{\text {tot }}(1.8 \mathrm{TeV})(\mathrm{mb})$ & $77.6_{-2.7}^{+2.5}$ & $76.8_{-2.7}^{+2.9}$ & $76.4_{-3.1}^{+3.4}$ \\
\hline$\sigma_{\text {tot }}(10 \mathrm{TeV})(\mathrm{mb})$ & $108.4_{-6.7}^{+7.0}$ & $106.4_{-6.7}^{+7.9}$ & $105.4_{-7.5}^{+9.2}$ \\
\hline$\sigma_{\text {tot }}(14 \mathrm{TeV})(\mathrm{mb})$ & $115.8_{-7.7}^{+8.3}$ & $113.5_{-7.8}^{+9.3}$ & $112.3_{-8.6}^{+10.8}$ \\
\hline
\end{tabular}

Table 4: Simple pole fit to total $p p$ and $\bar{p} p$ cross sections, and to the $\rho$ parameter, with non-degenerate $C=+1$ and $C=-1$ meson exchanges.

\begin{tabular}{|c|c|c|c|}
\hline parameter & all data & filtered data $(2 \sigma)$ & filtered data $(1 \sigma)$ \\
\hline$\chi^{2}$ & 197.9 & 107.7 & 56.3 \\
$\chi^{2}$ per d.o.f. & 1.39 & 0.82 & 0.52 \\
\hline pomeron intercept-1 & $0.0955_{-0.0083}^{+0.0097}$ & $0.0940_{-0.0079}^{+0.0092}$ & $0.095_{-0.010}^{+0.013}$ \\
pomeron coupling $(\mathrm{mb})$ & $18.4_{-2.0}^{+1.8}$ & $18.8_{-2.0}^{+1.7}$ & $18.5_{-2.6}^{+2.1}$ \\
\hline$\rho / \omega$ intercept -1 & $-0.535_{-0.059}^{+0.051}$ & $-0.518_{-0.058}^{+0.050}$ & $-0.540_{-0.007}^{+0.059}$ \\
$\rho / \omega$ coupling $C_{-}(p p)(\mathrm{mb})$ & $-31.6_{-11.5}^{+7.6}$ & $-28.9_{-10.4}^{+6.8}$ & $-32.5_{-13.9}^{+8.8}$ \\
\hline$a / f$ intercept -1 & $-0.338_{-0.055}^{+0.054}$ & $-0.355_{-0.057}^{+0.056}$ & $-0.346_{-0.066}^{+0.067}$ \\
$a / f$ coupling $(\mathrm{mb})$ & $58.8_{-6.8}^{+8.7}$ & $61.5_{-7.7}^{+9.8}$ & $60.4_{-7.9}^{+10.5}$ \\
\hline$\sigma_{\text {tot }}(1.8 \mathrm{TeV})(\mathrm{mb})$ & $77.3_{-2.7}^{+2.6}$ & $77.2 \pm 2.6$ & $77.2_{-3.3}^{+3.6}$ \\
\hline$\sigma_{\text {tot }}(10 \mathrm{TeV})(\mathrm{mb})$ & $106.8_{-6.3}^{+7.1}$ & $106.3_{-6.2}^{+6.8}$ & $106.5_{-7.8}^{+9.7}$ \\
\hline$\sigma_{\text {tot }}(14 \mathrm{TeV})(\mathrm{mb})$ & $113.9_{-7.4}^{+8.3}$ & $113.2_{-7.1}^{+8.0}$ & $113.5_{-9.0}^{+11.4}$ \\
\hline
\end{tabular}

Table 5: Simple pole fit to total $p p$ and $\bar{p} p$ cross sections, and to the $\rho$ parameter, with non-degenerate $C=+1$ and $C=-1$ meson exchanges, and using the alternative data set of Ref. [7].

In order to understand better the treatment of the errors, we give in Table 5 the result of a fit to the data of Ref. [7], where the errors have been added in quadrature. We see that the results are very stable, especially those 
for the pomeron intercept. This is similar to the statement that the filtering of the data does not affect the parameters very much.

At this point, the only additional piece of data might be the direct observation of the pomeron, i.e. of a $2^{++}$glueball. The WA91 collaboration has indeed confirmed [13] the WA71 observation of the $\mathrm{X}(1900)$ and showed that it was a $I^{G} J^{P C}=0^{+} 2^{++}$state, $f_{2}(1900)$. Its mass has been observed to be $1918 \pm 12 \mathrm{MeV}$. If we assume that this is the first state on the pomeron trajectory, and use the determination $\alpha^{\prime}=0.250 \mathrm{GeV}^{-2}$ [细, we obtain $\alpha_{P}=1.0803 \pm 0.012$. This is the value of the intercept for 1 -pomeron exchange. The intercepts that we obtained in Tables 1, 3, 4 and 5 from scattering data cannot be directly compared with this value, as they include the effect of multiple exchanges, of pomerons and reggeons. But the values we have derived are certainly compatible with the WA91 measurement. Note however that the conversion of the glueball mass into a pomeron intercept relies heavily on the value of the pomeron slope. An intercept of 1.094 would be in perfect agreement with the WA91 observation for a slope $\alpha^{\prime}=0.246$ $\mathrm{GeV}^{-2}$. Hence it would be dangerous to mix this piece of information with the $t$-channel information. Our best estimate for the pomeron intercept is then:

$$
\alpha_{P}=1.0964_{-0.0091}^{+0.0115}
$$

based on the $2 \sigma$-filtered PDG data, in the non-degenerate case.

Finally, we can place constraints on physics beyond one-pomeron exchange. The first obvious correction at high energy has to be related to unitarisation. Clearly, multiple exchanges must tame the rise of total cross sections so that they eventually obey the Froissart-Martin bound. Hence one would expect the value of the intercept to be an effective one, which decreases as $\sqrt{s}$ increases. We have already seen in Fig. (1.b) that the data does not show any sign of unitarisation up to Tevatron energies. One can confirm this by introducing a 2-pomeron exchange term in the amplitude. Although little is known about such a contribution, there is general agreement that it must be negative, and have an intercept $2 \alpha_{P}-1$. We can then fit the data to a form:

$$
\operatorname{Im} \mathcal{A}(s, 0)=C_{-} s^{\alpha_{-}}+C_{+} s^{\alpha_{+}}+C_{P}\left(s^{\alpha_{P}}+R s^{\alpha_{2 P}}\right)
$$

with $\alpha_{2 P}=2 \alpha_{P}-1$. Using $2 \sigma$-filtered data, we then obtain an upper bound 
on the ratio $R$ of its coupling to that of the pomeron:

$$
\frac{\mid 2-\text { pomeron coupling } \mid}{1-\text { pomeron coupling }}<4.7 \% \quad(70 \% \text { C.L. })
$$

Including such a contribution would bring the best value for the intercept of the 1-pomeron exchange term to $1.126_{-0.082}^{+0.051}$.

By following the method of Eq. (8), one can obtain bounds on more exotic objects. At the $70 \%$ C.L., the ratio of the coupling of an odderon to that of a pomeron is smaller than $0.1 \%$ (the best odderon intercept would then be 1.105 and the pomeron intercept become 1.099). This would correspond to $0.08 \mathrm{mb}$ at the Tevatron. As for the "hard pomeron", there is no trace of it in the data. Constraining its intercept to being larger than 1.3 leads to an upper bound on the ratio of its coupling to that of the pomeron of $0.9 \%$ (the soft pomeron intercept then becomes 1.065). This would correspond to a maximum hard contribution of $19 \mathrm{mb}$ at the Tevatron.

In conclusion, we have shown that simple pole fits to total cross sections are very successful. We show in Fig. 5 the results obtained in this paper together with other estimates present in the literature.

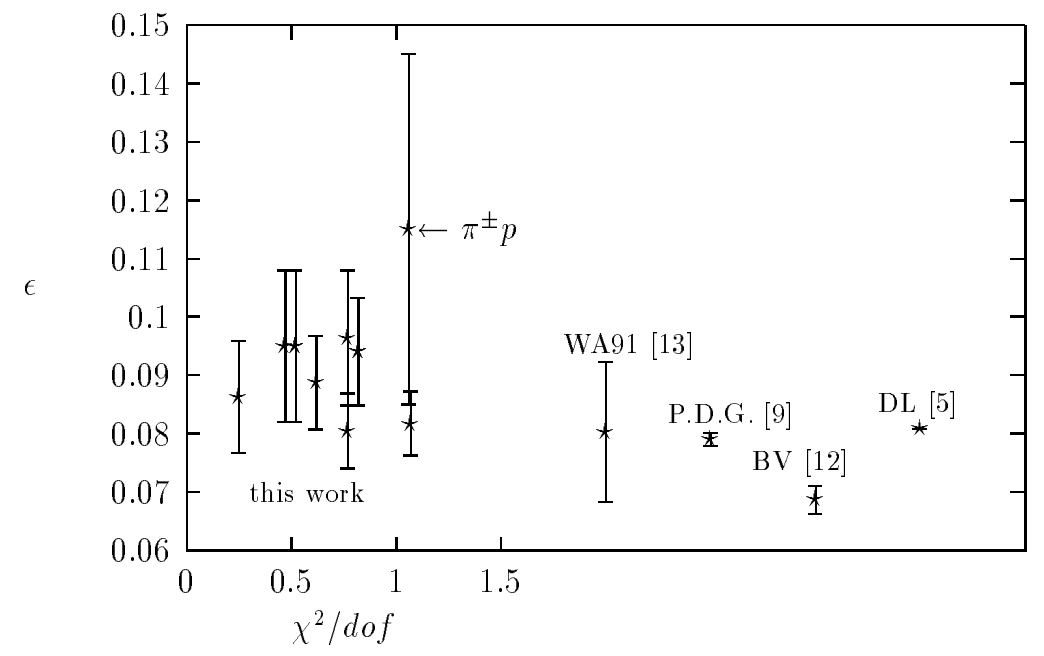

Figure 5: Our results for the pomeron intercept, compared with others in the literature. The values of the $\chi^{2} / d o f$ are indicated for the points of this work only. 
All the points from this work have an acceptable $\chi^{2}$, and the main difference between them is either the filtering of data or the physics of lower trajectories. Hence all these estimates are acceptable, and we feel that intercepts as high as 1.11, and as low as 1.07, are possible. When comparing with other work in the literature, we have explained that the use of a small energy cutoff leads to smaller intercepts, and reflects the fact that sub-leading meson trajectories are to be included [note that the original DL fit [5] used the same cutoff as ours, but used a different definition of $\chi^{2}$ [14]]. Our errors are much larger than those of other estimates because we fully take into account the correlation of the various parameters, and because our statistical analysis of the data is much more careful than previous ones. In other words, we quote the projection of the hypersurface containing $70 \%$ of the probability, rather than letting the $\chi^{2}$ simply vary by one unit. For this problem, we believe that this leads to much more reasonable error estimates, as explained above.

Note that these results depend only on $p p$ and $\bar{p} p$ data. We have argued that little could be learned from other hadronic reactions, given that they are measured at low energy. In particular, we want to point out that our fit to total cross sections of Fig. 3 is indistinguishable from the DL fit for $\sqrt{s}<300 \mathrm{GeV}$, hence the parametrisation we propose will fit the total $\gamma p$ cross section, as well as the $\pi p$ and $K p$ data.

\section{Acknowledgments}

We thank P.V. Landshoff for discussion and for sending us the data set used in the original DL fit. We are also grateful to P. Valin for his explanation of statistical subtleties. We also wish to acknowledge discussions with A. Burnel and P. Marage.

\section{References}

[1] I. Ya Pomeranchuk, Sov. Phys. JETP 34(7) (1958) 499.

[2] ZEUS Collaboration (M. Derrick et al.) Phys. Lett. B315 (1993), 481; H1 Collaboration (T. Ahmed et al.), Nucl. Phys. B429 (1994) 477. 
[3] Available at http://www-pdg.lbl.gov/xsect/contents.html, courtesy of A. Baldini, V. Flaminio, W.G. Moorhead, and D.R.O. Morrison, CERN; and COMPAS Group, IHEP, Protvino, Russia.

[4] A. Donnachie and P.V. Landshoff, Nucl. Phys. B244 (1984) 322; Nucl. Phys. B231 (1984) 189; Nucl. Phys. B267 (1986) 690.

[5] A. Donnachie and P.V. Landshoff, Phys. Lett. B296 (1992) 227, e-Print Archive: hep-ph/9209205.

[6] UA4.2 Collaboration, C. Augier et al., Phys Lett B (1993) 503.

[7] S. Hadjitheodoridis, Ph.D. Thesis, Brown University (May 1989).

[8] see e.g. K. Kang and S.K. Kim, preprint BROWN-HET-1008 (Oct. 1995), talk given at 7th Rencontres de Blois: Frontiers in Strong Interactions - 6th International Conference on Elastic and Diffractive Scattering, Blois, France, 20-24 June 1995, e-Print Archive: hep-ph/9510438; K. Kang, P. Valin and A.R. White, Nuov. Cim. 107A (1994) 2103.

[9] Review of Particle Physics, Particle Data Group, R.M. Barnett et al., Phys. Rev. D54 (1996).

[10] F. James, M. Roos Computer Physics Commun. 10 (1975) 343; F. James and M. Goosens, Minuit Reference Manual, CERN Program Library Long Writeup D506 (March 1994), available from ftp://asis01.cern.ch/cernlib/doc/ps.dir/minuit.ps.

[11] Numerical Recipes in C, William H. Press et al. (Cambridge University Press, Cambridge: 1988), Chapter 14.

[12] A. Bueno and J. Velasco, preprint IFIC-96-15 (May 1996).

[13] WA91 Collaboration, F. Antinori et al., Phys. Lett. B353 (1995) 589, WA76 Collaboration, T.A. Armstrong et al., Phys. Lett. B228 (1989) 536.

[14] P.V. Landshoff, private communication. 